\title{
La visibilité des territoires dans Geo et dans National Geographic Magazine (des années 1980 à la fin des années 2000)
}

Une ouverture sur le monde... et sur soi-même

The territorial visibility in Geo and National Geographic magazines (from the 1980's to the late 2000's): An opening to the world... and to oneself

A visibilidade dos territórios nas revistas Geo e National Geographic (da década de 1980 até o final da década de 2000): Uma abertura para o mundo... e para si mesmo

La visibilidad de los territorios en las revistas Geo y National Geographic (de la década de 1980 al final de la década de 2000): Una abertura al mundo... y a sí mismo

\section{Guilhem Labinal}

\section{OpenEdition}

Journals

Édition électronique

URL : https://journals.openedition.org/terrabrasilis/3012

DOI : 10.4000/terrabrasilis.3012

ISSN : 2316-7793

Éditeur

Rede Brasileira de História da Geografia e Geografia Histórica

Référence électronique

Guilhem Labinal, « La visibilité des territoires dans Geo et dans National Geographic Magazine (des années 1980 à la fin des années 2000) », Terra Brasilis [En ligne], 10 | 2018, mis en ligne le 26 décembre 2018, consulté le 05 décembre 2022. URL : http://journals.openedition.org/terrabrasilis/ 3012 ; DOI : https://doi.org/10.4000/terrabrasilis.3012

Ce document a été généré automatiquement le 5 décembre 2022

Tous droits réservés 


\title{
La visibilité des territoires dans Geo et dans National Geographic Magazine (des années 1980 à la fin des années 2000)
}

\author{
Une ouverture sur le monde... et sur soi-même \\ The territorial visibility in Geo and National Geographic magazines (from the \\ 1980's to the late 2000's): An opening to the world... and to oneself \\ A visibilidade dos territórios nas revistas Geo e National Geographic (da \\ década de 1980 até o final da década de 2000): Uma abertura para o mundo... e \\ para si mesmo \\ La visibilidad de los territorios en las revistas Geo y National Geographic (de \\ la década de 1980 al final de la década de 2000): Una abertura al mundo... y a sí \\ mismo
}

\section{Guilhem Labinal}

1 Dans le sillon de travaux réalisés sur les quotidiens nationaux dès les années 1960 pour tester, dans les médias, les déterminants de la représentation des États (Galtung et Ruge, 1965), de récentes recherches ont développé des outils en géographie pour mieux rendre compte de la visibilité des territoires dans l'environnement informationnel actuel (Grasland et al., 2016). Ainsi l'étude des flux RSS internationaux émis par divers quotidiens d'information (fichiers XML consultables par les internautes) atteste de ce que $20 \%$ des États assurent près de $80 \%$ des mentions (Beauguitte et al., 2016). Certains territoires sont peu couverts par les médias, des hiérarchies existent dans la presse et sur Internet. Concernant les contenus de la presse magazine et, notamment, les magazines plus anciens, les disparités observables constituent des indices exploitables pour mieux saisir la nature des représentations livrées aux lecteurs. C'est, du moins, ce que nous proposons de montrer à partir des index associés à deux 
publications d'importance, National Geographic Magazine et Geo. Nous le faisons en nous appuyant sur une thèse (Labinal, 2009) dont nous révisons certains résultats pour interroger, ici, la pertinence de l'examen de leur couverture spatiale à l'échelle étatique sur une trentaine d'années de parution (du début des années 1980 à la fin des années 2000). Un regard croisé sur ces deux mensuels, le premier dans sa version américaine et le second dans sa déclinaison française est d'autant plus intéressant que leur diffusion est restée conséquente malgré les difficultés rencontrées par la presse. Ce faisant, nous identifions les limites imposées par la convocation des index pour analyser la vision du monde véhiculée par ces magazines.

\section{Étudier les index à l'échelle étatique: une méthodologie... et ses limites}

2 Quels sont les pays les plus évoqués dans les magazines grand public de géographie? Les angles morts? Et en quoi la sélection témoigne-t-elle d'un projet, d'une stratégie de communication? Pour tenter d'apporter, dans cet article, quelques éléments de réponse à ces questions, nous nous inspirons de certains critères développés dans le cadre des Media studies par les spécialistes de la communication (par exemple: Wu 1998, 2000, 2003, 2007). Ces critères ont été utilisés ou discutés par les géographes dans le cadre, notamment, de l'ANR Corpus Géomédia ${ }^{1}$ ainsi que par d'autres chercheurs qui travaillent avec eux en s'intéressant à la dimension spatiale de la diffusion de l'information (en particulier: Severo, 2014). Nous nous rapprochons également des études portant sur l'imagerie construite par un magazine comme le National Geographic (Lutz et Collins, 1993; Beaudreau, 2002; García-Álvarez et Marías-Martínez, 2013) et notamment de celles qui ont identifié le caractère impérialiste de ses livraisons jusqu'aux années 1950 (Rothenberg 1993, 2007; Tuason, 1999; Hawkins, 2010). Pour appréhender les sur- et les sous-représentations spatiales dans une perspective critique nécessitant de vérifier certains contenus directement dans les dossiers des magazines, nous nous focalisons sur les index proposés par les concepteurs des publications de notre corpus: d'abord celui élaboré Geo, index alphabétique accessible en ligne ${ }^{2}$ qui autorise des recherches par destination, rubrique, auteur ou mot clé et qui recense les articles publiés depuis la création du support; ensuite celui proposé dans le coffret édité à l'occasion des 125 ans du National Geographic Magazine, ${ }^{3}$ lequel permet une recherche similaire dès lors qu'y sont retenues certaines options. A l'issue des premières expérimentations, nous avons délaissé l'index directement consultable sur le portail du National Geographic ${ }^{4} \mathrm{du}$ fait du manque de lisibilité de ses principes de recherche et, surtout, de la diversité des publications pour lesquelles les résultats sont susceptibles de faire écho, l'emblématique magazine disposant de nombreuses déclinaisons intégrées à la base de données (National Geographic Kids magazine, Traveler magazine, ouvrages divers...).

3 Comme les options de sélection sont plurielles dans les index, nous avons privilégié une modalité de recherche à l'échelle étatique favorisant des résultats similaires entre les deux magazines de l'étude. La fonction de recherche proposée par The Complete National Geographic, une fois sélectionnés les filtres pertinents, ${ }^{5}$ rend effectivement comparable la sélection obtenue avec celle proposée par l'index de Geo si l'on retient les "petits" et les "grands" dossiers. Nous avons exclu les mentions diverses (dans une illustration par exemple) car la pertinence d'un tel suivi apparait discutable lorsque l'on travaille à 
l'échelle mondiale au vu de la diversité des erreurs potentielles pour certains pays et de la lourdeur des vérifications nécessaires (on compte parfois plus de 2000 références). En effet, au-delà d'une possible critique épistémologique qui pourrait porter sur la focale étatique retenue dans la mesure où celle-ci ne prend pas en charge la diversité des thèmes susceptibles de rendre compte, en géographie, de la spatialité d'un territoire sans pour autant le mentionner, plusieurs limites sont rapidement identifiables. Les premières sont d'ordre historique du fait des modifications territoriales qui rendent complexe la recherche des dénominations plus anciennes. La situation de l'ex U.R.S.S. impose à l'analyste, par exemple, la prudence sur les conclusions portant sur son aire d'extension et, plus largement, les régions qui ont éprouvé des bouleversements géopolitiques importants font question.

4 Les limitations de l'exploitation du corpus sont ainsi imputables à des facteurs divers, d'ordre générique (liées à la forme) ou génétique (liées au fond) comme le cas des territoires homonymes en témoigne: la Géorgie est référencée à la fois comme un territoire européen autonome et, dans le même temps, comme un État fédéré du sudest des États-Unis ou comme un territoire insulaire situé dans l'Océan Atlantique. Dans ce cas, la limite est également d'ordre scalaire: on relèvera plus particulièrement le fait que plusieurs territoires sont abordés par le biais d'une région, d'une ville ou d'un espace à l'échelle infranationale et qu'ils peuvent être ignorés dans l'index dans la mesure où ils ne sont pas rapportés à l'État dans lequel ils s'insèrent. L'Abkhazie par exemple, indépendante depuis sa sécession avec la Géorgie en 1992 mais dont le statut d'État n'est reconnu que par quelques pays, n'apparaît qu'une fois dans Geo alors que plusieurs articles antérieurs évoquent la Géorgie et, parfois, l'Abkhazie à travers le Caucase. En outre, l'analyse à l'échelle étatique ne permet pas de saisir les disparités qui se font jour à plus grande échelle: il suffit, pour s'en convaincre, d'observer celles repérables dans la représentation des États brésiliens dans le mensuel américain lorsque l'on procède à une recherche fine de l'ensemble des mentions (Figure 1). 
Figure 1. La représentation des États brésiliens dans National Geographic Magazine (1979-2009)

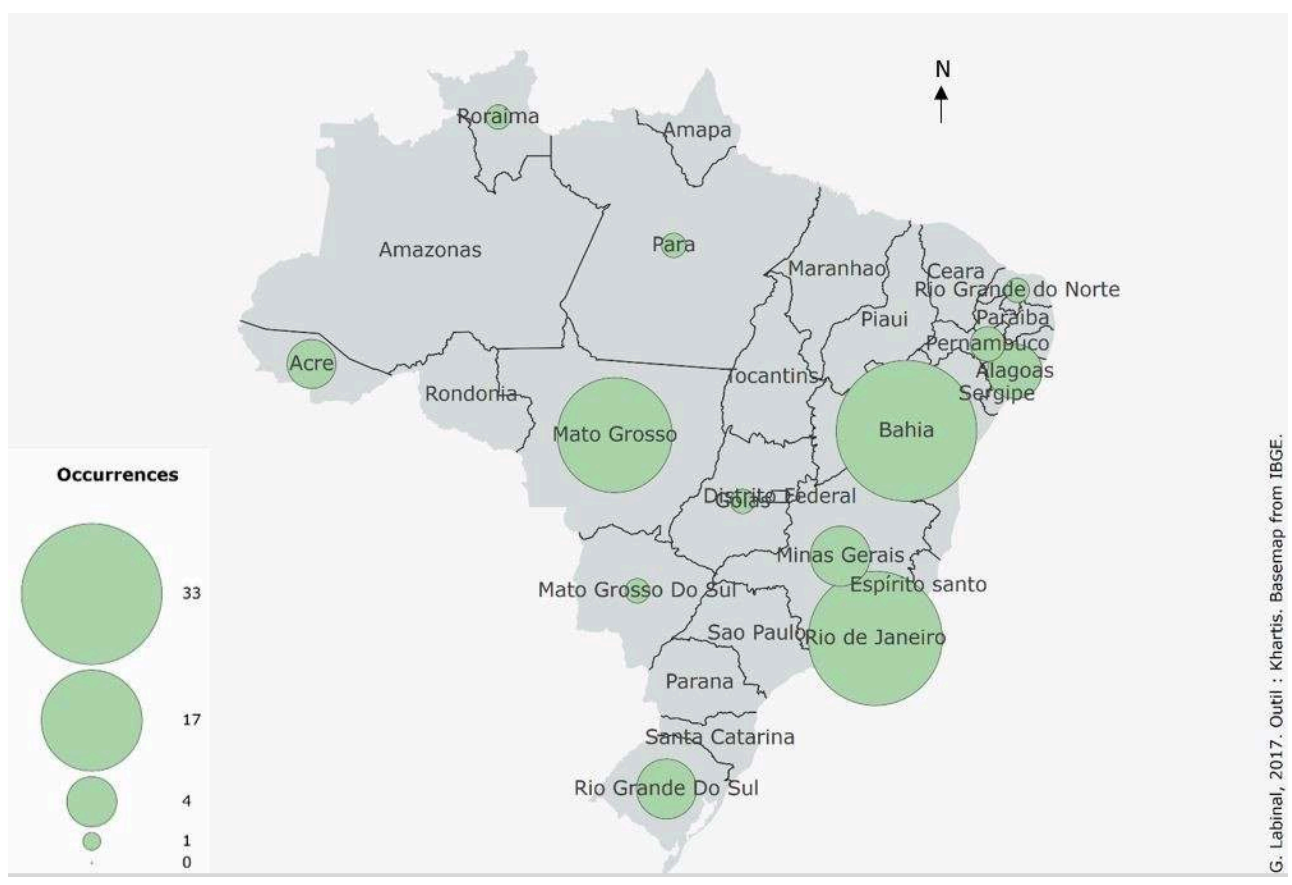

Certains États, tels Tocantins ou Maranhão, ne font l'objet d'aucune mention. Les États plus urbains, bordiers de l'Atlantique sont privilégiés de même que les États dont l'exploitation accrue des terres a renforcé l'accessibilité (le Mato Grosso par exemple) alors que les espaces plus enclavés de l'Amazonie apparaissent délaissés. La très grande majorité des reportages portant sur les fronts pionniers se concentrent sur la disparition de la faune, de la flore et de la forêt dense ombrophile imputable à la progression des terres cultivées ou à la surexploitation. Les États du Sud et du Sudeste bénéficient d'un plus grand nombre de reportages et d'un traitement diversifié (sur l'espace urbain et les favelas, sur certains quartiers touristiques, sur l'activité touristique associée aux plages et sur la musique, le carnaval, etc.) et, dans le Nordeste, c'est Bahia qui bénéficie d'un traitement particulier parce que l'État est traité, notamment, à travers des thématiques culturelles et historiques liées à l'esclavage et au passé colonial.

Données de la carte obtenues à partir d'une recherche de l'ensemble des mentions - sujets de dossiers, cartes, illustrations, etc. - des différents États (toutes ont été vérifiées par une consultation systématique des magazines).

Source: Guilhem Labinal

De façon générale, l'ambiguïté de certaines requêtes est évidente (le cas de la République du Congo - le Congo Brazzaville - et de la République démocratique du Congo - le Congo Kinshasa - suffisent à s'en convaincre) et il convient de rester prudent lorsque l'on examine la couverture spatiale de tel ou tel magazine à partir de son index. Par exemple, dans celui de National Geographic Magazine, la sélection d'options multiples pour examiner les mentions de tel ou tel État - et non plus les dossiers portant sur eux ou dans lesquels ils occuperaient une place centrale - rend les résultats discutables. Citons le cas d'Antigua-et-Barbuda, plus facile à cerner du fait du nombre limité d'occurrences qui le caractérisent. Identifier les mentions de ce territoire dans les illustrations ou dans d'autres contenus sans se focaliser sur les dossiers portant directement sur cet espace nécessite, pour être complet, d'effectuer la requête "Antigua" car le territoire est aussi désigné de cette façon par le magazine (sous l'appellatif "Antigua Island" par exemple). Toutefois, alors que l'index recense 14 mentions de cet État, seules 4 d'entre elles apparaissent pertinentes: certaines concernent une municipalité de l'État de Veracruz au Mexique ("La Antigua") quand d'autres désignent "Antigua Guatemala", l'ancienne capitale du Royaume du Guatemala... Et les cas sont nombreux; paradoxalement, le Royaume-Uni n'est gratifié 
que de 181 mentions quand l'Angleterre bénéficie de 1036 occurrences sur la période sondée... Le problème pourrait trouver, certes, une partie de sa solution dans sa propre formulation, dans la mesure où le fait de relever les occurrences sur trente ans de publication pourrait favoriser un relevé suffisamment conséquent pour dégager des tendances. Mais, en définitive, élargir la recherche à l'ensemble des citations reste périlleux car les mentions erronées, les doublons ou les faux négatifs sont difficiles à cerner sur des résultats si nombreux. Faudrait-il pour autant se garder d'exploiter l'ensemble des données proposées dans l'index? Ce serait là une erreur car se centrer sur les dossiers et non sur les mentions diverses, plus délicates à vérifier, autorise un recueil fructueux des sujets et des dossiers dans lesquels chaque territoire est mentionné: la restriction du champ de la recherche permet effectivement une évaluation des occurrences si l'on applique des correctifs, pour limiter les biais, en consultant directement les magazines quand la situation l'impose.

\section{De nombreux angles morts...}

63610 occurrences territoriales ont été relevées dans Geo pour 1359 dans National Geographic Magazine sur une période peu ou prou identique. ${ }^{6}$ Le rapport est donc plus de deux fois et demi supérieur dans la première pour un nombre de pages légèrement inférieur par numéro: on y verra, pour l'essentiel, la diversification des sujets dont National Geographic Magazine est coutumier alors que les dossiers de Geo prennent plus systématiquement en charge une certaine forme de spatialité (l'analyse sur 101 mois de publication à partir des index imprimés avait révélé, il y a quelques années, un rapport comparable). ${ }^{7}$ Mais cet écart n'enlève rien à l'utilité d'une comparaison: simplement, le relevé des occurrences donne, en premier lieu, un aperçu de la visibilité des territoires dans chacun des deux magazines.

7 La lecture des données témoigne d'abord de ce que l'ancrage spatial des dossiers de Geo privilégie les pays du Nord au détriment de ceux du Sud, suivant un modèle gravitaire de régulation de l'information (centre/périphérie) identifié de longue date (Galtung, 1971). Les pays de l'Europe de l'Est (Russie incluse) sont peu représentés; la difficulté, pour les journalistes occidentaux, d'y accéder durant la période de la Guerre froide y est sans doute pour beaucoup. Ces territoires représentent 4,35\% du quantum d'occurrences des territoires par rapport à l'ensemble de ceux cités dans National Geographic Magazine, 3,30\% des occurrences dans Geo. Si l'on exclut la Russie, la proportion baisse substantiellement: de plus d'un point et demi dans les deux cas. Certains territoires ne sont jamais mentionnés par ailleurs: un micro-État comme Andorre, par exemple, ne fait l'objet d'aucun sujet, de même que certains territoires insulaires totalement absents de ces deux magazines (Saint-Kitts-et-Nevis, SaintVincent, Barbade, Nauru). Plus largement, si les reportages s'intéressant à des pays touristiques comme le Pérou abondent, un grand nombre de pays moins estimés des agences de voyages sont peu ou pas évoqués - à l'image de l'Uruguay qui, pendant trois décennies, n'a fait l'objet d'aucun sujet particulier dans National Geographic Magazine pour un seul dans Geo.

8 Enfin, à plus petite échelle, il apparait qu'au-delà de l'Europe de l'Est, l'espace le plus délaissé sur les trois décennies reste celui de l'Asie centrale (Figure 1). Les sujets portant sur lui représentant moins de $2 \%$ de l'ensemble des dossiers référencés même si cette rareté est un peu plus marquée dans Geo que dans National Geographic Magazine. 
Plus qu'un strict désintérêt, la difficulté d'accès aux anciennes Républiques soviétiques pour les occidentaux constitue, ici, l'hypothèse la plus évidente pour comprendre le faible nombre de dossiers portant sur ces territoires dont la fixation des frontières, en 1991, a révélé de nombreux désaccords (Thorez, 2011). Pour d'autres motifs, l'Afrique centrale ou méridionale fait aussi moins souvent l'objet de mentions (à peine plus de 5,5\% de l'ensemble des dossiers dans les deux cas). Plus largement, des travaux ont montré que la couverture médiatique dont a bénéficié l'Afrique a été faible dans les médias américains malgré la lourdeur des difficultés rencontrées par certains pays (Golan, 2008). Dans notre corpus, les mentions de l'Afrique centrale ou méridionale sont très réduites comparativement aux territoires bordiers de la Méditerranée, ceux du Maghreb en particulier; l'Egypte se situe en bonne place dans l'index des deux magazines ( $11^{\mathrm{e}_{\mathrm{me}}}$ et $12^{\mathrm{ème}}$ rang des pays faisant l'objet du plus grand nombre de sujets): sans surprise ici, les destinations privilégiées par les touristes sont à l'honneur, un tel favoritisme pouvant paraître logique dans la mesure où la ligne éditoriale d'un magazine comme Geo le rattache à la presse de voyage (Figure 2 et Figure 3).

Figure 2. Graphique de la distribution spatiale des reportages de Geo $(1979$ - 2012) et de National Geographic Magazine (1979 à 2009)

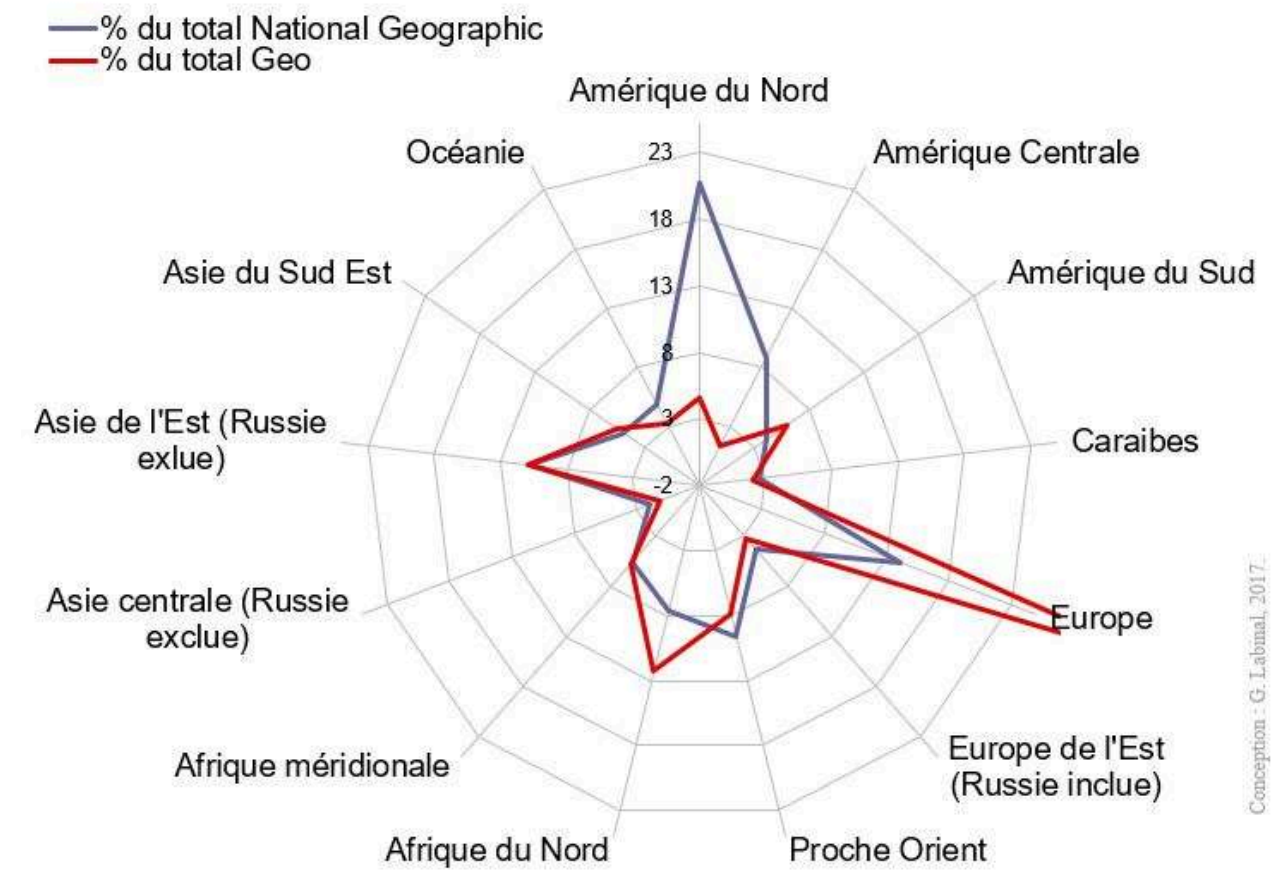


Figure 3. Carte de la distribution spatiale des reportages de Geo et du National Geographic France (de 1979 à 2012 pour Geo, de 1979 à 2009 pour National Geographic Magazine)

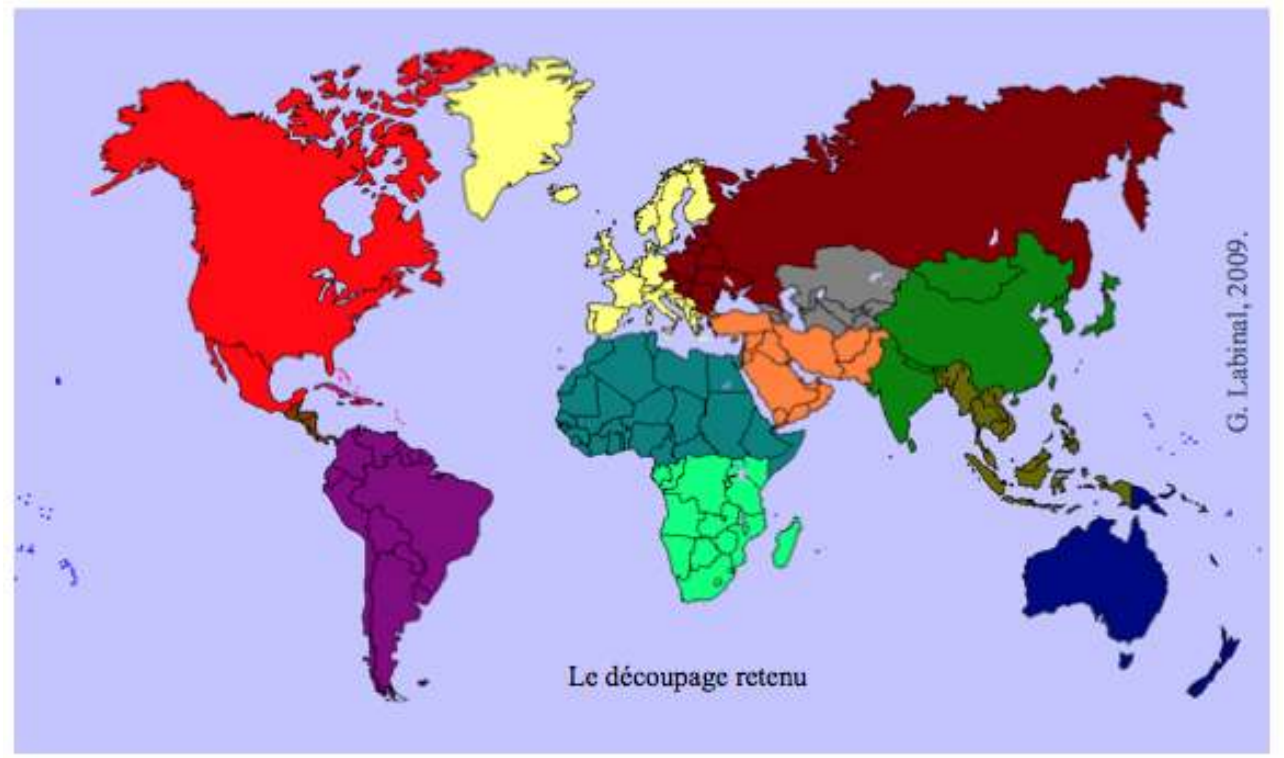

Source: Guilhem Labinal

\section{Des disparités importantes, des éléments traditionnels d'explication}

Les principes retenus par la cartographie qui suit prennent en compte les disparités observées. Répondre aux exigences d'une distribution dissymétrique des occurrences relevées à partir des index est ainsi nécessaire. Dans ce cadre, la constitution de classes d'effectifs égaux permet d'équilibrer la répartition des pays et, surtout, de favoriser la comparaison des séries relatives aux deux magazines (Figure 4 et Figure 5). ${ }^{8}$ Cinq pays bénéficient du quart des citations dans National Geographic Magazine quand cette proportion s'élève à près d'un tiers dans Geo. ${ }^{9}$ La concentration des sujets y est plus grande mais Geo couvre mieux l'espace mondial que National Geographic Magazine dont les angles morts sont nombreux (trois fois plus). ${ }^{10}$

10 La place accordée à la France dans Geo est plus importante que celle concédée aux ÉtatsUnis dans le magazine de la Society. Les lecteurs peuvent mesurer cette tendance comme en témoigne ce courrier de lectrice adressé à Geo:

Votre magazine présente beaucoup d'intérêt, mais je souhaite faire une petite observation sur les sujets traités. Est-ce pour flatter notre chauvinisme national ou attirer les touristes étrangers, je l'ignore, mais des articles très fréquents sur les régions françaises m'étonnent. Alors que l'Union européenne cherche à rapprocher les Etats qui en font partie, ce qui implique une meilleure connaissance de ces pays, il me semble que GEO pourrait consacrer plus souvent des articles à d'autres régions européennes que nos belles provinces françaises. ${ }^{11}$

11 L'omniprésence des reportages axés sur la France dans ce mensuel est patente, son espace métropolitain focalisant l'objet à lui seul $20 \%$ des sujets traités. Certes, d'autres territoires sont sur-représentés dans le magazine, mais ils le sont dans une moindre mesure. Le calcul d'un indice confrontant le premier et le second pays le plus évoqué par Geo établit, en effet, un rapport approximatif de 1 à 5,5 (entre la France et l'Italie) 
alors qu'il n'est que de 1 à 2 dans National Geographic Magazine (entre les États-Unis et le Honduras). Si cette dernière publication est moins centrée sur les États-Unis que Geo ne l'est sur la France, elle se focalise néanmoins de façon nette sur les pays de l'espace nord-américain: on enregistre plus de $20 \%$ de citations pour les États-Unis, le Canada et le Mexique. Ainsi, comme l'a montré l'étude des items de flux internationaux de 37 quotidiens récents qui a permis d'identifier une sur-représentation systématique des États voisins liée à la logique journalistique du "mort-kilomètre" (Beauguitte et al., 2016), l'effet de voisinage joue à plein; la proxémique explique sans doute cette répartition d'intérêts. ${ }^{12}$ La puissance des relations commerciales entretenues entre les pays voisins y est probablement pour beaucoup. La cartographie qui suit ne prend pas en considération le rapport entre le nombre d'occurrences, la superficie et/ou la population des territoires concernés mais il est clair qu'à l'exception des États dont l'immensité et le poids économique, démographique ou diplomatique justifient des reportages sur tous types de sujets (Inde, Chine, Russie, Japon...), ${ }^{13}$ la couverture spatiale des magazines parait autocentrée. Le Honduras est plus cité que l'Inde dans National Geographic Magazine et, dans Geo, l'Italie est plus citée que la Chine.

Figure 4. La couverture spatiale de National Geographic Magazine (1979-2009)

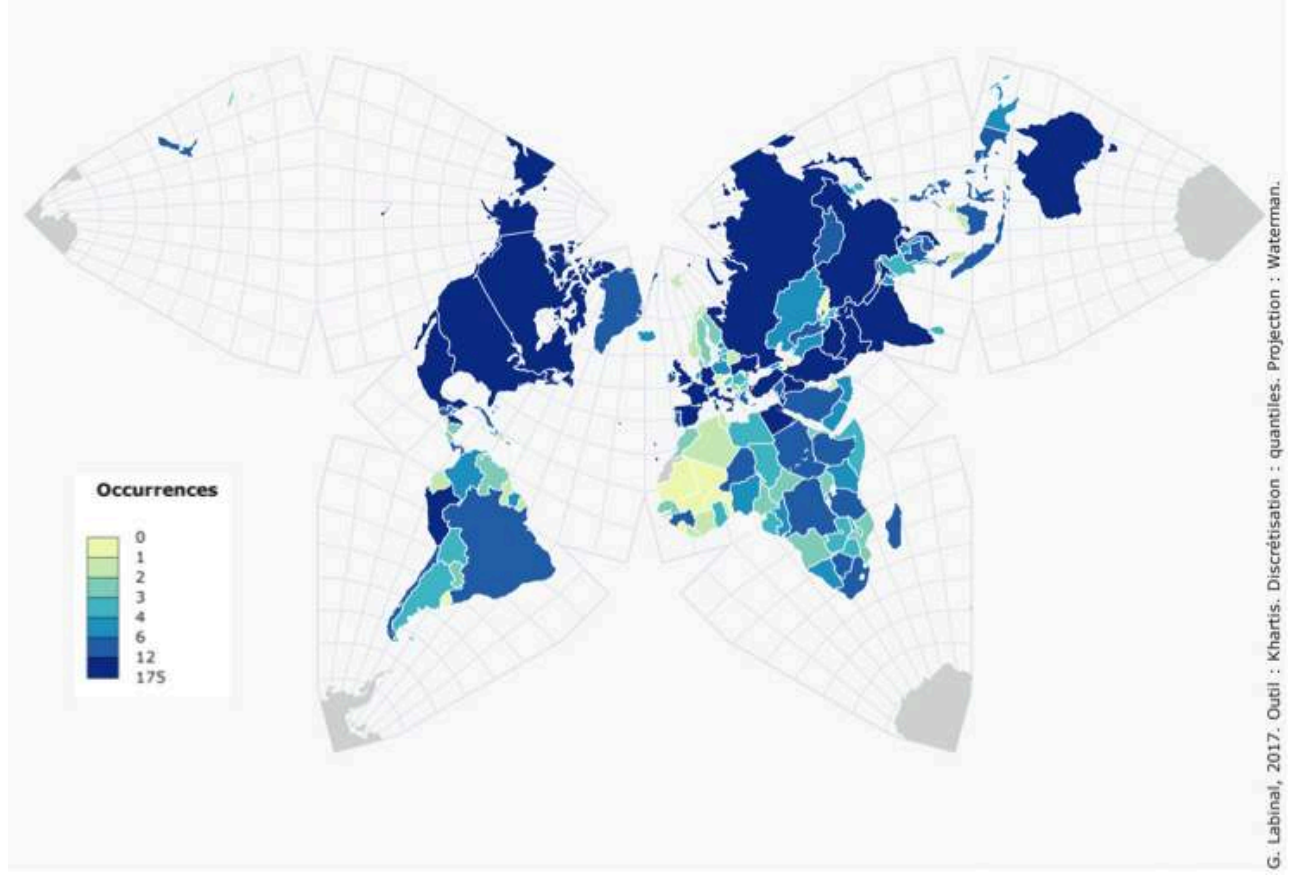

Source: Guilhem Labinal 


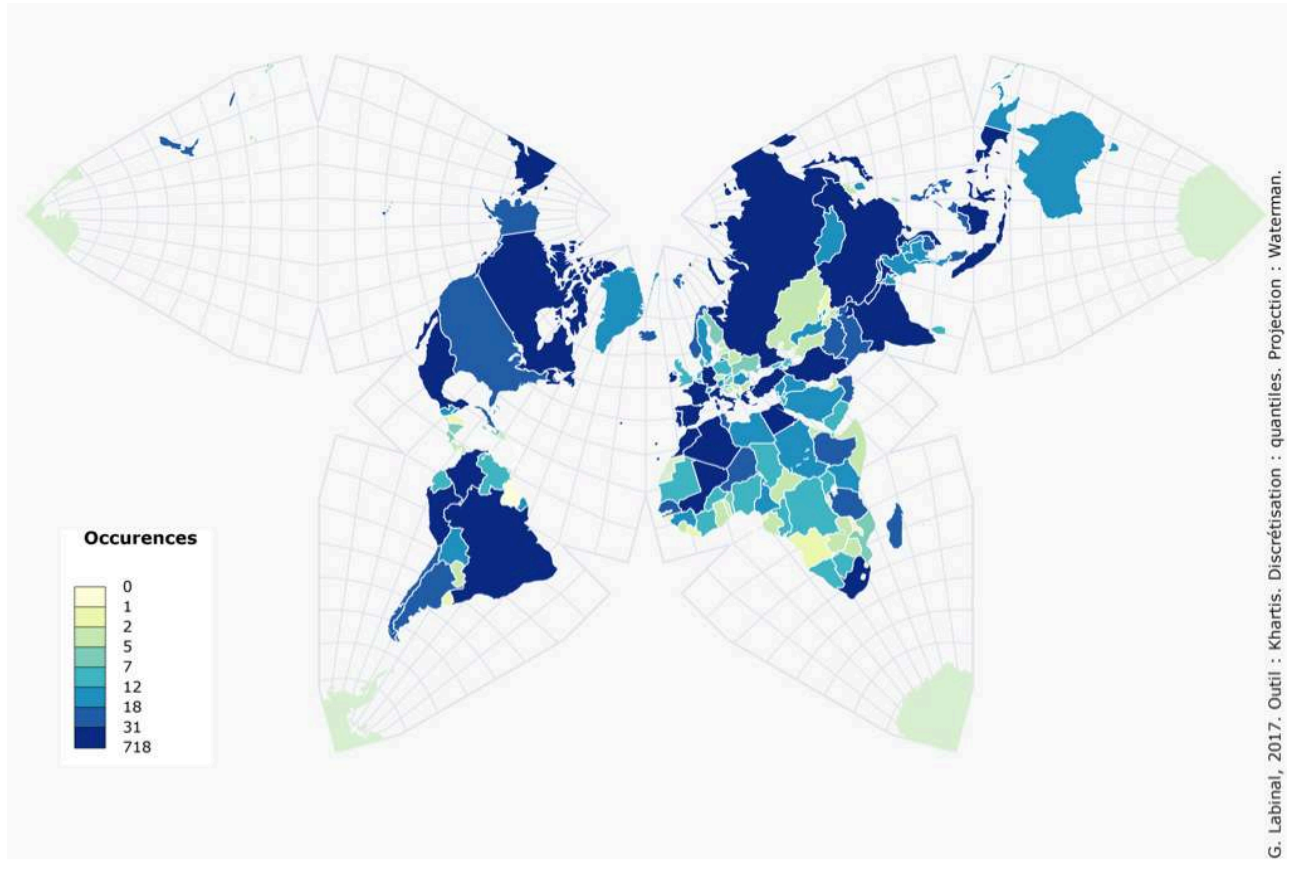

Source: Guilhem Labinal un tropisme touristique particulièrement marqué dans Geo et assumé, finalement, par la ligne éditoriale du magazine. En juillet 2008, ce dernier accompagnait ainsi sa livraison d'une publicité pour Nouvelles Frontières, soutenant une sélection de voyages choisis par le tour operator parce qu'elle lui semblait proche de l'authenticité à laquelle la ligne éditoriale souscrivait pour voyager dans de bonnes conditions. A tout le moins, c'est ce qu'une lettre publicitaire affirmait juste avant d'inviter les lecteurs à visiter le Cambodge, l'Inde ou encore l'Egypte - ce "pays des dieux":

(...) GEO et Nouvelles Frontières s'engagent dans un tourisme responsable, conscients de l'environnement mais surtout du respect des populations locales. Voilà pourquoi GEO parraine les itinéraires de Nouvelles Frontières qui répondent à l'esprit du magazine: hors des sentiers battus, riches en émotion, avec une approche authentique au contact de la nature et des peuples. ${ }^{14}$

Au-delà des lieux les plus fréquentés par les touristes, ceux qui sont habituellement privilégiés par la presse d'information quotidienne pour des raisons politiques sont privilégiés. L'île de Cuba, très présente dans les médias en raison de son rôle durant la Guerre froide, de la personnalité charismatique (et polémique) de Fidel Castro ou bien du camp de Guantanamo est le premier des espaces insulaires caribéens auxquels se sont intéressés les magazines. La Bosnie-Herzégovine fait partie des pays les plus évoqués par National Geographic Magazine et, sur la période étudiée, Geo a consacré près de 36 sujets à l'Iran dont la situation est relayée depuis la révolution islamique et la guerre qui a opposé Khomeiny à l'Iraq de Saddam Hussein. Le magazine a développé les différentes situations conflictuelles de ce pays même s'il ne s'est pas limité aux questions politiques, diplomatiques et religieuses. Les regards convergent souvent vers les "points chauds" du globe.

14 Toutefois, si le tourisme ou l'actualité internationale expliquent partiellement les choix réalisés, ils n'épuisent pas la diversité des motivations qui conduisent les magazines à 
cibler tel ou tel territoire. Certaines problématiques agissent comme des prismes mobilisés à l'envie pour évoquer un espace que l'on peine à envisager sous un angle différent: songeons, par exemple, au Congo évoqué de nombreuses fois à travers le travail de Jane Godall et les recherches menées sur les gorilles "en danger" dans National Geographic Magazine. ${ }^{15}$ L'observation de la couverture spatiale des deux publications que nous étudions résulte aussi de telles entrées, indifférentes aux facteurs prédictifs les plus souvent mis en avant pour rendre compte de l'inégale visibilité des territoires dans les médias.

\section{Un monde centré sur soi}

National Geographic Magazine accorde une place très importante aux sujets sur l'Amérique centrale. L'influence persistante de la distance sur la couverture des nouvelles étrangères a été établie pour les flux de nouvelles internationales (Koopmans et Vliegenthart, 2010). Faudrait-il voir en outre, dans la couverture spatiale du magazine américain, le révélateur d'un caractère post-impérialiste décrié, souvent avec raison, pour ce magazine? Au Guatemala, les reportages traitent dans une majorité de cas de l'univers archéologique des Mayas ${ }^{16}$ et, au Pérou, de l'univers Inca, de ses sépultures ou de celles des Mochicas. ${ }^{17}$ Ces sujets sont peu porteurs d'expression idéologique mais plusieurs pays auxquels le magazine s'intéresse particulièrement, comme le Mexique (Tableau 1), bénéficient de développements d'une toute autre nature. Le rapport affectif et matériel établi avec certains territoires de l'Amérique latine ou centrale s'est doublé, en effet, d'une relation d'utilité établie de longue date. On pourrait certes imaginer que les réminiscences de la doctrine Monroe ne sont pas étrangères à l'attention que porte le magazine à ces pays; un tel intérêt témoignerait, en effet, des traces laissées par ses principes dans les relations économiques et diplomatiques des États-Unis..$^{18}$ Pourtant, à mieux y regarder, la proportion des sujets portant sur l'Amérique du Sud est supérieure dans Geo et c'est bien ce dernier magazine qui réserve aux pays d'Amérique centrale un sort réduit à une peau de chagrin (la proportion étant à peine supérieure à celle dévolue à l'Asie centrale dans les deux mensuels). ${ }^{19}$ En changeant d'échelle d'observation, on note même une inégalité de traitement des pays à l'intérieur de l'aire sud-américaine: quand certains territoires concentrent toutes les attentions (le Pérou, le Brésil ou la Colombie), d'autres apparaissent très peu dans l'index des reportages.

Tableau 1. Pays les plus abordés dans Geo et dans National Geographic France

\begin{tabular}{|l|l|l|l|l|l|l|}
\hline \multicolumn{2}{|l|}{ National Geographic Magazine (1979-2009) } & \multicolumn{2}{l|}{ Geo (1979-2012) } \\
\hline Pays & $\begin{array}{l}\text { Occurrences/ } \\
\text { dossiers }\end{array}$ & $\begin{array}{l}\text { Part du } \\
\text { total (\%) }\end{array}$ & Pays & $\begin{array}{l}\text { Occurrences/ } \\
\text { dossiers }\end{array}$ & $\begin{array}{l}\text { Part du } \\
\text { total (\%) }\end{array}$ \\
\hline États-Unis & 175 & 12,88 & France & 718 & 19,89 \\
\hline Honduras & 96 & 7,06 & Italie & 131 & 3,63 \\
\hline Chine & 59 & 4,34 & Inde & 131 & 3,63 \\
\hline
\end{tabular}




\begin{tabular}{|c|c|c|c|c|c|}
\hline Canada & 55 & 4,05 & Chine & 112 & 3,10 \\
\hline Mexique & 50 & 3,68 & Canada & 100 & 2,77 \\
\hline Australie & 41 & 3,02 & Espagne & 84 & 2,33 \\
\hline Inde & 36 & 2,65 & Egypte & 79 & 2,19 \\
\hline Egypte & 34 & 2,50 & Japon & 70 & 1,94 \\
\hline $\begin{array}{l}\text { Bosnie- } \\
\text { Herzégovine }\end{array}$ & 31 & 2,28 & Russie & 54 & 1,50 \\
\hline Royaume-Uni & 25 & 1,84 & Mexique & 48 & 1,33 \\
\hline Russie & 21 & 1,55 & Maroc & 45 & 1,25 \\
\hline France & 18 & 1,32 & $\begin{array}{l}\text { Afrique du } \\
\text { Sud }\end{array}$ & 42 & 1,16 \\
\hline Japon & 18 & 1,32 & Pérou & 41 & 1,14 \\
\hline Viêt-Nam & 18 & 1,32 & Turquie & 41 & 1,14 \\
\hline Pérou & 17 & 1,25 & Algérie & 41 & 1,14 \\
\hline
\end{tabular}

Les recherches sur l'actualité internationale ont mis en évidence le rôle des liens culturels pour expliquer la visibilité des territoires dans les médias: l'étude du nombre d'occurrences des flux RSS australiens dessinent, par exemple, un monde orienté par un "effet Commonwealth" (Beauguitte et al., 2016: 137). Dans la version française du mensuel Geo, les territoires les plus abordés sont souvent très évocateurs pour une population dont est issue une grande partie de l'équipe éditoriale. ${ }^{20}$ Les pays rattachés à l'ex-empire colonial français - avec lesquels ont été conservés des liens affectifs puissants et des relations diplomatiques complexes (ceux de l'ancienne A.O.F, par exemple) - sont plus cités que les États qui se trouvaient sous mandats étrangers. Les dossiers sur la Libye sont ainsi trois à quatre fois moins nombreux que ceux portant l'Algérie ou le Maroc. La vision du monde véhiculée par le magazine est orientée par cet ancrage spatial sélectif qui témoigne des legs du mouvement colonial sans pour autant être réductible à sa matrice; le dynamisme des échanges commerciaux entretenus par la France avec d'autres territoires reste un facteur clé de compréhension, les deux aspects - culturel et économique - étant généralement liés. Le constat interpelle néanmoins d'autant plus que le système de références de Geo est centré sur la France. L'ancrage spatial en appelle, semble-t-il, à un imaginaire balisé et domestiqué par la culture des journalistes et du public ciblé. Un imaginaire qui s'intéresse à l'autre mais qui renvoie d'abord à soi-même.

National Geographic Magazine cherche à s'éloigner du voyage touristique moins porteur de cet imaginaire romantique, entre science et art, qu'il suscitait déjà grâce à ses photographies à la fin du siècle dernier (Hawkins, 2010). Le magazine propose des entrées à caractère ethnographique permettant, aux lecteurs, de s'immerger dans les espaces sur lesquels il leur offre un certain regard. Souvenons-nous qu'aux États-Unis, 
le premier numéro du National Geographic était apparu bien après que soit effectué durant la décennie 1840 - le "basculement de la figure du touriste" identifié par Sylvain Venayre (2004): un basculement qui en fit "un voyageur sans aventures, arpentant un espace qui avait cessé d'être un espace lointain". ${ }^{21}$ La publication américaine s'est développée dans ce que l'historien a défini comme un "temps de regret" caractérisé par une nostalgie imputable au rétrécissement du monde..$^{22} C^{\prime}$ 'est là une différence, sans doute, avec Geo qui développe le voyage plus que l'exploration même si, dans les deux cas, le lecteur est amené à élaborer un imaginaire fondé sur une projection indissociable de ses propres références. Le commentaire laissé sur le site du journal français Le Monde au sujet des images proposées par le National Geographic Magazine illustre la demande à laquelle le mensuel américain tente ainsi de répondre:

Voyager sur la terre va devenir de plus en plus monotone et sans charme; la Chine détruit à tour de bras son patrimoine d'exotisme, l'Arabie devient une galerie marchande de luxe. Seuls les voyages dans le temps, en images et imagination, resteront une source de rêves et de poésie. Racontez-moi comment était le monde autrefois, comment étaient les voyages...merci pour les images du monde. ${ }^{23}$

18 Ainsi cette nostalgie, vivace, est actualisée sans cesse par la publication américaine: elle fait du déplacement (culturel, intellectuel) la condition d'un éloignement dans le temps et, pour le dire autrement, la possibilité de retrouver le sentiment qui accompagnait les explorations du passé. En se rapprochant parfois de la littérature illustrée du XIXe siècle et, par-là, de l'héritage impérialiste européen, les choix du magazine ont pu être très orientés politiquement comme en attestent les analyses critiques qui ont porté sur ses publications antérieures (Rothenberg 1993, 2007; Hawkins, 2010). La spatialité des médias est liée aux enjeux de pouvoir et au rôle des agences de presse; en ce qui concerne la presse américaine, on sait depuis longtemps que le poids démographique, économique ou militaire des pays dans la hiérarchie des nations est un facteur essentiel pour expliquer la représentation différenciée des États (Robinson et Sparkes, 1976; Rosengren, 1977; Kim et Barnett, 1996). Mais, de même que l'analyse des flux émis par des journaux édités dans différents pays en attestent (Gasher et Gabriele, 2004), d'autres facteurs entrent en considération car les stratégies des médias dépendent aussi des attentes supposées du public auquel ils s'adressent.

\section{Conclusion}

19 Si la cartographie des données contenues dans les index permet de mettre en évidence des sur- ou des sous-représentations spatiales intéressantes, c'est ajoutée à la façon dont les territoires sont appréhendés, au rôle qui leur est conféré qu'elle peut contribuer à éclairer plus finement le système de représentations par lequel un magazine tend à se singulariser. Quand le corpus est circonscrit, travailler sur les index des magazines imprimés contraint, autant que nécessaire, à se confronter aux contenus des dossiers. Cependant, en dépit des précautions qui s'imposent, la couverture spatiale des magazines en témoigne: le poids économique ou politique, le développement touristique ou l'inscription dans l'actualité internationale des territoires rendent compte très classiquement de l'inégale visibilité des pays dans les publications étudiées. Alors que l'on aurait pu supposer un rôle très limité de l'effet de distance pour des magazines qui proposent, à leurs lecteurs, de se dépayser, ce dernier conditionne leur couverture spatiale de façon manifeste, à l'image de ce qui a pu être établi pour certains quotidiens d'information nationaux (Koopmans et Vliegenthart, 2010). Une 
distance spatiale, donc, mais aussi culturelle car la dimension autoréférentielle du dépaysement proposé est certaine. Le monde de Geo et de National Geographic Magazine est adapté à la spécificité du public ciblé pour satisfaire ses attentes supposées. Conformément à ce que Rogers (1995) a nommé une "exposition sélective", les magazines évitent les messages qui pourraient entrer "en conflit" avec les prédispositions de leurs lecteurs. ${ }^{24}$ Ainsi répondent-ils à ce qui est conçu, sans doute, comme un impératif nécessaire à leur diffusion dans un cadre concurrentiel.

\section{BIBLIOGRAPHIE}

Beaudreau, Sylvie (2002). "The changing faces of Canada: images of Canada in National Geographic”. The American Review of Canadian Studies, v. 32, n. 4 (Winter), pp. 517-546.

Beauguitte, Laurent; Severo, Marta; Pécout, Hugues (2016). “Une analyse géomédiatique de l'actualité internationale: hiérarchies et effets de voisinage". L'Espace géographique, 2016/2 (Tome 45), pp. 124-141.

Beauguitte, Laurent; Severo, Marta; Pécout, Hugues (2016). L'actualité internationale: entre hiérarchie globale et stratégies éditoriales régionalisées". CIST2016 En quête de territoire(s)?, Proceedings du 3 e colloque international du CIST, pp. 59-65.

Galtung, Johan; Ruge, Mari Holmboe (1965). "The Structure of Foreign News. The Presentation of the Congo, Cuba and Cyprus Crises in Four Norwegian Newspapers". Journal of peace research, v. 2, n. 1, pp. 64-90.

Galtung, Johan. (1971). “A structural theory of imperialism”. Journal of Peace Research, v. 8, n. 2, pp. 81-118.

García-Álvares, Jacobo; Marías-Martínez, Daniel (2013). “Geographical magazines and popular geographies: the case of the Revista Geográfica Española, 1938-1977”. Journal of Historical Geography, v. 39, pp. 85-98.

Gasher, Mike; Gabriele, Sandra (2004). "Increasing Circulation? A Comparative News-Flow Study of the Montreal Gazette's Hard-Copy and On-Line Editions". Journalism Studies, v. 5, n. 3, pp. 311-312.

Golan, Guy (2008). "Where in the world is Africa? Predicting coverage of Africa by U.S. television networks”. International Communication Gazette, v. 70, n. 1, pp. 41-57.

Grasland, Claude; Lamarche-Perrin, Robin; Loveluck, Benjamin; Hugues, Pecout (2016). "Vers une modélisation de l'agenda géomatique international: distribution spatiale, temporelle et médiatique des flux d'actualité à travers le monde". L'Espace géographique, Éditions Belin, 2016/1, pp. 25-43.

Hawkins, Stephanie (2010). American Iconographic: National Geographic, Global Culture, and the Visual Imagination. Charlottesville, University of Virginia Press, $252 \mathrm{p}$. 
Jones, Timothy; Van Aelst, Peter; Vliegenthart, Rens (2013). "Foreign Nation Visibility in U.S. News Coverage: A Longitudinal Analysis (1950-2006)”. Communication Research, v. 40, n. 3, pp. 417-436. Première publication en ligne: 19 juillet 2011.

Kim, Kyungmo; Barnett, George (1996). "The determinants of international news flow: A network analysis". Communication Research, v. 23, n. 3, pp. 323-352.

Koopmans, Ruud; Vliegenthart, Rens (2010). "Media attention as the outcome of a diffusion process - A theoretical framework and cross-national evidence on earthquake coverage". European Sociological Review, v. 27, n. 5, pp. 636-653. Première publication en ligne: doi:10.1093/ esr/jcq032

Labinal, Guilhem (2009). La géographie des médias. Une analyse iconologique et textuelle des magazines. Thèse de doctorat de géographie, Université Paris 1.503 p.

Lutz, Catherine; Collins, Jane (1993). Reading national geographic, Chicago, University of Chicago Press, $309 \mathrm{p}$.

Robinson, Gertrude Joch; Sparkes, Vernone (1976). "International news in the Canadian and American press: Comparative news flow study”. International Communication Gazette, v. 22, n. 4, pp. 203-218.

Rogers, Everett (1995). Diffusion of Innovations. New York: Free Press, 538 p. 1ère éd., 1962.

Rosengren Karl Erik (1977). “Four types of tables”. Journal of Communication, v. 27, n. 1, pp. 67-75.

Rothenberg, Tamar (1993). "Voyeurs of Imperialism: The National Geographic before World War Two”. In: Godlewska, Anne; Smith, Neil. (Eds.), Geography and Empire: critical studies in the history of geography, Oxford, Blackwell, 1993, pp. 155-172.

Rothenberg, Tamar (2007). Presenting America's World: Strategies of innocence in National Geographic Magazine, 1888-1945. Hampshire: Ashgate, 202 p.

Severo, Marta (2014). "L'information quotidienne face au Web 2.0. La stratégie multiplateforme de six quotidiens nationaux français". Études de communication, v. 41, n. 2, pp.89-102.

Thorez, Julien (2011), “Les nouvelles frontières de l'Asie centrale: États, nations et régions en recomposition”. Cybergeo: European Journal of Geography [En ligne], Politique, Culture, Représentations, document 534, mis en ligne le 25 mai 2011, consulté le 10 juillet 2017. URL: http://cybergeo.revues.org/23707; DOI: 10.4000/cybergeo.23707

Tuason, Julie (1999). “The ideology of empire in National Geographic magazine's coverage of the Philippines, 1898-1908”. Geographical Review, v. 89, n. 1, pp. 34-53.

Venayre, Sylvain. (2004). “Qu'est-ce que l'éloignement ? L'aventure, l'ethnographie et les blancs de la carte (1850-1940)”. In: Laboulais-Lesage, Isabelle (Dir.), Combler les blancs de la carte. Modalités et enjeux de la construction des savoirs géographiques (XVIe-XXe siècle). Études réunies avec le concours de Jean-François Chauvard et Odile Goerg, Presses Universitaires de Strasbourg, pp. 61-87.

Wu, Haoming Denis (1998). "Investigating the determinants of international news flow: A metaanalysis". International Journal for Communication Studies, v. 60, n. 6, pp. 493-512.

Wu, Haoming Denis (2000). "Systemic determinants of international news coverage: A comparison of 38 countries". Journal of Communication, v. 50, $n^{\circ} 2$, pp. 110-130.

Wu, Haoming Denis (2003). 'Homogeneity around the world?: Comparing the systemic determinants of international news flow between developed and developing countries". 
International Communication Gazette, Los Angeles, London, New Delhi and Singapore, v. 65, n. 1, pp. 9-24.

Wu, Haoming Denis (2007). "A brave new world for International news?. Exploring the Determinants of the Coverage of Foreign Nations on US Websites". The International Communication Gazette, Los Angeles, London, New Delhi and Singapore, v. 69, n. 6, pp. 539-555.

\section{NOTES}

1. Le but du projet était de mener une recherche conjointe, "à la fois géographique et médiatique, sur les échanges contemporains de flux d'information médiatique entre les pays du Monde". Cf. Edition 2011 du programme Corpus, données et outils de la recherche en sciences humaines et sociales. Consultable sur Internet (au 7 octobre 2010). URL: <https://geomedia.hypotheses.org/>.

2. Index consultable sur le site du magazine (consulté le 23 mai 2017). Cf. URL: <http:// www.geo.fr/index-magazines-geo>.

3. National Geographic (2013). The Complete National Geographic. Every Issue Since 1888 of National Geographic Magazine on Your Computer. NG Society, 7 DVD-ROMs.

4. Cf. National Geographic Publications Index. URL: <http://shadow.ngs.org/Search/default.aspx? $c t x=5 \cdot 1033 \cdot 0.0 .3>$.

5. Les options que nous avons sélectionnées dans les index sont les suivantes : articles de fond (feature articles), cartes en suppléments - lesquelles constituent souvent des sortes de dossiers complémentaires.

6. Les années 1979-2012 ont été prises en considération dans le premier cas tandis que l'examen s'arrête à décembre 2009 dans le second.

7. Le décompte avait été opéré en observant les numéros publiés de mai 1999 à septembre 2007 pour le magazine Geo, d'octobre 1999 à février 2008 pour le National Geographic France. Il avait permis d'identifier, ponctuellement, quelques erreurs: par exemple, dans l'ancien index du National Geographic France, un numéro évoquant Salvador de Bahia ( $\mathrm{n}^{\circ} 35$, août 2002) avait été mis au compte du Salvador alors qu'il devait créditer le Brésil.

8. Il s'agit de la méthode par quantiles. Nous avons utilisé ici le logiciel Khartis mis au point par l'atelier cartographique de Sciences Po. URL: <http://www.sciencespo.fr/cartographie/khartis/>.

9. L'ensemble est constitué des États-Unis, du Honduras, de la Chine, du Canada et du Mexique dans le National Geographic Magazine (1979-2009), de la France, de l'Italie, de l'Inde, de la Chine et du Canada dans Geo (1979-2012).

10. En effet, seuls 13 États ne font l'objet d'aucun sujet dans Geo alors qu'ils sont 36 à ne pas retenir l'attention de National Geographic Magazine.

11. Courrier de Mme Lamblet (Dijon) publié dans la livraison de septembre 2005. La réponse du magazine à cette observation prend la forme d'une justification: "Nous nous sommes régulièrement intéressés à nos voisins européens, anciens, nouveaux et futurs, avec des reportages sur la Pologne, les pays Baltes, la Croatie, un Spécial Europe, etc. Mais réjouissez-vous puisque notre prochain grand dossier sera consacré à l'Europe sauvage, ses espaces naturels et, notamment, ses grands carnivores" (Geo, septembre 2005, n³19, p. 8).

12. Grâce à Abraham Moles, on sait désormais l'intérêt de "l'étude de l'ensemble des faits vitaux dans lesquels, ce qui est proche est pour moi (ici, maintenant) plus important que ce qui est lointain (ailleurs, autrefois, plus tard)". Cf. Moles, A. (1995), "Vers une psycho-géographie". In: Bailly, A.; Ferras, R.; Pumain, D. L'Encyclopédie de la Géographie. Paris, Economica, p. 159.

13. C'est ce que tendent à démontrer les études portant sur les quotidiens américains d'information. C'est le cas, notamment, de celle de H. D. Wu (2007, p. 547) même si la puissance politique (la présence au conseil de sécurité de l'ONU, la capacité nucléaire...) est un déterminant 
plus grand que le poids économique pour certains médias tel le New York Times (à la différence de NBC), ainsi que l'ont montré d'autres recherches par ailleurs (Jones et al., 2013).

14. Réclame jointe à la livraison des abonnés du magazine Geo en juin 2008 (La Sicile et les îles Eoliennes, $\mathrm{n}^{\mathrm{0}}$ 353). Les passages en gras sont surlignés dans le texte original.

15. On citera, à titre d'exemple, l'article "En compagnie des géants" qui se fixe sur le "triangle de Djéké", dans le nord du Congo (National Geographic France, n 100, janvier 2008, pp. 36-53).

16. Consulter les livraisons du magazine National Geographic France d'avril 2002 ( $\mathrm{n}^{\circ} 31$ ), d'août $2003\left(n^{\circ} 47\right)$ ou encore de novembre $2004\left(n^{\circ} 62\right)$.

17. Cf. National Geographic France $n^{\circ} 18$ (mars 2001), $n^{\circ} 48$ (septembre 2003), $n^{\circ} 57$ (juin 2004), $n^{\circ} 71$ (août 2005).

18. Les principes énoncés par Monroe devant le Congrès (2 décembre 1823) avaient défini cette partie du monde comme une chasse gardée pour les États-Unis qui, par-là, répondaient aux mouvements d'indépendance des pays d'Amérique latine (1818-1823). Ils avaient ouvert la voie de l'impérialisme américain : leur application a ensuite permis aux Etats-Unis de prendre l'avantage sur l'Espagne dans la guerre qui les a opposés en 1898. Le champ libre leur fut laissé pour annexer Porto-Rico puis pour promouvoir un amendement donnant à Washington la possibilité d'intervenir à Cuba en 1903 (l'île n'obtint son indépendance qu'avec la victoire de F. Castro en 1959).

19. Selon ce décompte réalisé sur 101 mois (de mai 1999 à septembre 2007 pour Geo, d'octobre 1999 à février 2008 pour le National Geogaphic France): l'Asie centrale - Russie exclue - mobilise seulement $1,83 \%$ des reportages de Geo et $1,92 \%$ de ceux de l'édition française du National Geographic.

20. Quelques nuances éditoriales seraient toutefois à observer entre les différentes versions de Geo, les équipes éditoriales étrangères semblant bénéficier d'une certaine latitude par rapport à l'équipe initiale (par exemple, la version publiée à Singapour: Asian GEOgraphic).

21. Venayre, S. (2004). Qu'est-ce que l'éloignement ? L'aventure, l'ethnographie et les blancs de la carte (1850-1940). In: Laboulais-Lesage (sous la dir. de). Combler les blancs de la carte. Modalités et enjeux de la construction des savoirs géographiques (XVIe-XXe siècle), Études réunies avec le concours de Jean-François Chauvard et Odile Goerg, Presses Universitaires de Strasbourg, p. 73.

22. Ibidem, p. 75.

23. Commentaire laissé le 15 février 2013 à 12 h 49 sous le pseudonyme "M F" en réponse à la mise en ligne du dossier portfolio "National Geographic", 125 ans d'images par le journal Le Monde (le 24.01.2013 à $15 \mathrm{~h} 12$ et mis à jour le 24.01.2013 à 17h14). Cf. URL: <http:// www.lemonde.fr/planete/portfolio/2013/01/24/national-geographic-125-ans-d-

images_1821237_3244.html\#i8KgHA54HvbtoqqH.99>.

24. E. M. Rogers precise à propos de ce qu'il a nommé "selective explosure": "Individuals tend to expose themselves to ideas that are in accordance with their interests, needs, and existing attitudes. Individuals consciously or unconsciously avoid messages that are in conflict with their predispositions" - cf. Rogers, E. (1995). Diffusion of Innovations, New York: Free Press, p. 164. 1ère éd., 1962. Cité par Koopmans, R.; Vliegenthart, R. (2010). Media attention as the outcome of a diffusion process - A theoretical framework and cross-national evidence on earthquake coverage. European Sociological Review, v. 27/5, p. 5. 


\section{RÉSUMÉS}

Les analyses des flux RSS internationaux émis sur Internet par les grands quotidiens d'information établissent une forte concentration de l'actualité, certains pays étant très peu couverts par les médias. Si l'on s'intéresse aux magazines grand public de géographie pour analyser leur couverture spatiale sur plusieurs décennies, un examen des index conçus par les éditeurs apparaît pertinent pour mettre au jour les hiérarchies. L'objectif de cet article est donc d'identifier la couverture spatiale de National Geographic Magazine et de Geo sur une trentaine d'années de parution - du début des années 1980 à la fin des années 2000 - en interrogeant les limites associées à la convocation des index à l'échelle étatique. Des réserves méthodologiques doivent être soulignées, mais l'examen du corpus confirment les analyses réalisées sur d'autres types de supports. Le développement touristique, l'actualité internationale ou la puissance des États éclairent les choix des magazines et les legs historiques, la proximité culturelle et l'effet de voisinage justifient également les sélections: le lecteur est amené à élaborer un imaginaire fondé sur une projection indissociable de ses propres références.

Recent research analyzing the international RSS feeds of multiple dailies clearly demonstrates a high concentration in international news. Geographical coverage is uneven. If the interest is focused on popular geographical magazines to analyze hierarchies on several decades, it becomes relevant to study the indexes complied by the publishers. In this article, our aim is therefore to evaluate the spatial coverage of National Geographic Magazine and Geo over a period of about thirty years, from the 1980 s to the late 2000s, by asking ourselves where are the limitations of using indexes on a state level. Boundaries should be drawn to ensure our conclusions but an examination of data obtained confirms the findings of Media studies that went before. It reveals that even if spatial coverage can be partially explained by tourism development, current international events or power of states, the spatial coverage is also justified by historical and political legacies, cultural proximities and neighborhoods. Readers are put in the position to promote an individual's world view based on their own references.

Pesquisas recentes que analisam os fluxos internacionais de RSS de vários noticários demonstram claramente uma alta concentração de notícias internacionais. A cobertura geográfica das notícias é desigual. Se há um interesse em analisar a cobertura espacial de revistas populares de geografia ao longo de várias décadas, pareceu-nos relevante a elaboração de uma revisão dos indicadores projetados por editores para revelar hierarquias nessa cobertura. O objetivo deste artigo é, portanto, identificar a abrangência espacial da revista National Geographic Magazine e da Geo ao longo de trinta anos de publicação, desde o início da década de 1980 até o final da década de 2000, perguntando-nos quais são as limitações no uso de indicadores no nível do Estado-Nação. Limites devem ser traçados para garantir nossas conclusões, mas um exame dos dados obtidos confirma a descoberta de estudos de mídia (media studies) anteriores. Tais estudos indicam que, mesmo que a cobertura espacial possa ser explicada pelo desenvolvimento do turismo, as notícias internacionais ou o poder dos Estados também informam a escolha das revistas e do seu consequente legado histórico. Proximidade cultural e feito de vizinhança também justificam as seleções. Em síntese, os leitores são levados a desenvolver um imaginário baseado em uma projeção inseperável de suas próprias referências.

Los analisis del flujo internacional de RSS emitidos en internet por los grandes diarios de información establecen una fuerte concentración de las noticias, algunos países siendo muy poco cubiertos por dichos medios. Al interesarse por las revistas de geografía dirigidas al gran público para analizar su cobertura espacial a lo largo de varios decenios, un examen de los índices 
elaborados por los editores parece pertinente para establecer las jerarquías. El objetivo de este artículo es de esta manera el de identificar la cobertura espacial de National Geographic Magazine y Geo a lo largo de una treintena de años de publicación - desde el comienzo de los años de 1980 hasta el fin de la década de 2000-, interrogando los límites asociados a la organización de los índices en escala estatal. Reservas metodológicas deben resaltarse, sin embargo el examen del corpus confirma los análisis realizados sobre otro de soportes. El desarrollo turístico, la actualidad internacional y el poder de los Estados aclaran las elecciones de las revistas y los legados históricos, al tiempo que la proximidad cultural y el efecto de vecindad justifican igualmente las selecciones: el lector es conducido a elaborar un imaginario fundado en una proyección indisociable de sus propias referencias.

\section{INDEX}

Index géographique : Mundo

Palavras-chave : Revistas geográficas populares, mídia, visão de mundo, imperialismo

Palabras claves : revistas geográficas populares, medios, visión de mundo, imperialismo Index chronologique : $1980-2000$

Mots-clés : magazines grand public de géographie, médias, vision du monde, impérialisme

Keywords : popular geographical magazines, media, worldview, imperialism

\section{AUTEUR}

\section{GUILHEM LABINAL}

Maître de conférences en géographie/Assistant Professor, ÉSPÉ - Université de Cergy-Pontoise. Laboratoire EMA (École, Mutations, Apprentissages), UCP. Chercheur associé à l'UMR 8504 Géographie-cités - CNRS, équipe E.H.GO. Bureau C 235, ZAC des Barbanniers, Avenue Marcel Paul, 92230 Gennevilliers. guilhem.labinal@u-cergy.fr 